\title{
Preventive, personalized medicine at the level of key regulatory proteins: updates on activity- dependent neuroprotective protein (ADNP) as a case study
}

\author{
Illana Gozes \\ From EPMA-World Congress 2013 \\ Brussels, Belgium. 20-21 September 2013
}

Natural ageing, neurodegenerative diseases, acute head (brain) injury and stroke as well as mental disorders are associated with nerve cell damage and death. We have discovered activity-dependent neurotrophic factor (ADNP) that is essential for brain formation, regulating $>400$ important genes, including the major risk gene for Alzheimer's disease, apolipoprotein E. ADNP constitutes a part of complex essential for proper gene activity [1]. In men, the ADNP gene is mutated in autism, deregulated in terms of brain expression in schizophrenia [2] and significantly reduced in the blood of patients with neurodegenerative disease such as Alzheimer's disease [3] and multiple sclerosis. New results indicate the potential of ADNP monitoring as indicator for disease progression, paving the path to preventive treatment with ADNP replacement therapies. In this respect, the ADNP peptide derivative NAP (davunetide) has shown neuroprotection in multiple animal models of neurodegeneration (protecting against axonal transport deficits) [4] as well as in a recent schizophrenia clinical trial [5]. It is thus of major interest to further investigate ADNP as a predictive marker and ADNP replacement as a personalized, preventive, protecting therapy against neurodegeneration.

\section{Support}

AMN Foundation, Adams Super Center for Brain Studies, Canadian Friends of Tel Aviv University, Montreal Circle of Friends and the Adams Family.
Published: 11 February 2014

\section{References}

1. Dresner EMalishkevich A, Arviv C, Leibman Barak S, Alon S, Ofir R, Gothilf Y, Gozes I: Novel evolutionary-conserved role for the activity-dependent neuroprotective protein (ADNP) family that is important for erythropoiesis. J Biol Chem 2012, 287:40173-40185.

2. Dresner E, Agam G, Gozes I: Activity-dependent neuroprotective protein (ADNP) expression level is correlated with the expression of the sister protein ADNP2: deregulation in schizophrenia. Eur Neuropsychopharmacol 2011, 21:355-361.

3. Yang $M H$, Yang YH, Lu CY, Jong SB, Chen LJ, Lin YF, Wu SJ, Chu PY, Chung TW, Tyan YC: Activity-dependent neuroprotector homeobox protein: A candidate protein identified in serum as diagnostic biomarker for Alzheimer's disease. J Proteomics 2012, 75:3617-3629.

4. Jouroukhin Y, Ostritsky R, Assaf Y, Pelled G, Giladi E, Gozes I: NAP (davunetide) modifies disease progression in a mouse model of severe neurodegeneration: protection against impairments in axonal transportation. Neurobiol Dis 2013, 56:79-94.

5. Jarskog LF, Dong Z, Kangarlu A, Colibazzi T, Girgis RR, Kegeles LS, Barch DM, Buchanan RW, Csernansky JG, Goff DC, Harms MP, Javitt DC, Keefe RS, McEvoy JP, McMahon RP, Marder SR, Peterson BS, Lieberman JA: Effects of davenetide on $\mathrm{N}$-acetylaspartate and choline in dorsolateral prefrontal cortex in patients with schizophrenia. Neuropsychopharmacology 2013, 38:1245-1252.

\section{doi:10.1186/1878-5085-5-S1-A97}

Cite this article as: Gozes: Preventive, personalized medicine at the level of key regulatory proteins: updates on activity-dependent neuroprotective protein (ADNP) as a case study. EPMA Journal 2014 5(Suppl 1):A97.

\footnotetext{
Correspondence: ygozes@gmail.com

The Adams Super Center for Brain Studies; The Lily and Avraham Gildor Chair for the Investigation of Growth Factors; The Elton Laboratory for Neuroendocrinology; Department of Human Molecular Genetics and Biochemistry, Sagol School of Neuroscience, Sackler Faculty of Medicine, Tel Aviv University, Tel Aviv 69978, Israel
} 